\title{
Demand Analysis of Starchy Food Staples in Uganda Using Micro-data
}

\author{
Gilbert J. Werema \\ Associate Professor of Economics \\ College of Business \\ Texas Woman's University \\ P.O. Box 425738 \\ Denton, TX 76204-5738, USA
}

\begin{abstract}
This paper evaluates the consumption of 14 food groups, focusing on starchy staple foods and using the LA/AIDS framework using the Uganda National Household Survey of 2000. In particular, the LA/AIDS models are estimated to calculate income and price elasticities for three different components of food categories. We found that urban families consume more matooke, sugar, other cereals, oils, fruits and vegetables, fish, dairy products, other foods, and pulses than their counterparts in the rural areas. Households located in border districts more likely purchase maize, matooke, and meat than those in non-border areas. At lower incomes, price changes results in greater consumer substitution within the starchy food groups.
\end{abstract}

JEL classifications: C31, D10, D11, D12, Q10, Q18

Keywords: Food, Income, Price, Demand, Food Policy, Linear Almost Ideal Demand System, Uncompensated elasticity, Compensated elasticity.

\section{Introduction}

In many poor countries, cereals and roots provide two-thirds or more of the total calorie intake. Uganda, being a poor country, is no exception and exhibits this trend. According to Figure 1, which shows the share of food consumption between 1990 and 1994, 51\% of calorie intake in Uganda came from cereals and roots. Figure 2 confirms this high share of consumption of cereals by showing the highest consumed cereals in Uganda being millet, maize, sorghum, and rice, respectively. Teuteberg (1975) showed that, as incomes rose in Western Europe at the turn of this century, the proportions of calories from cereal and roots declined. This is a trend we are expecting to see in the case Uganda.

Figure 1: UGANDA: Share of Food Consumption

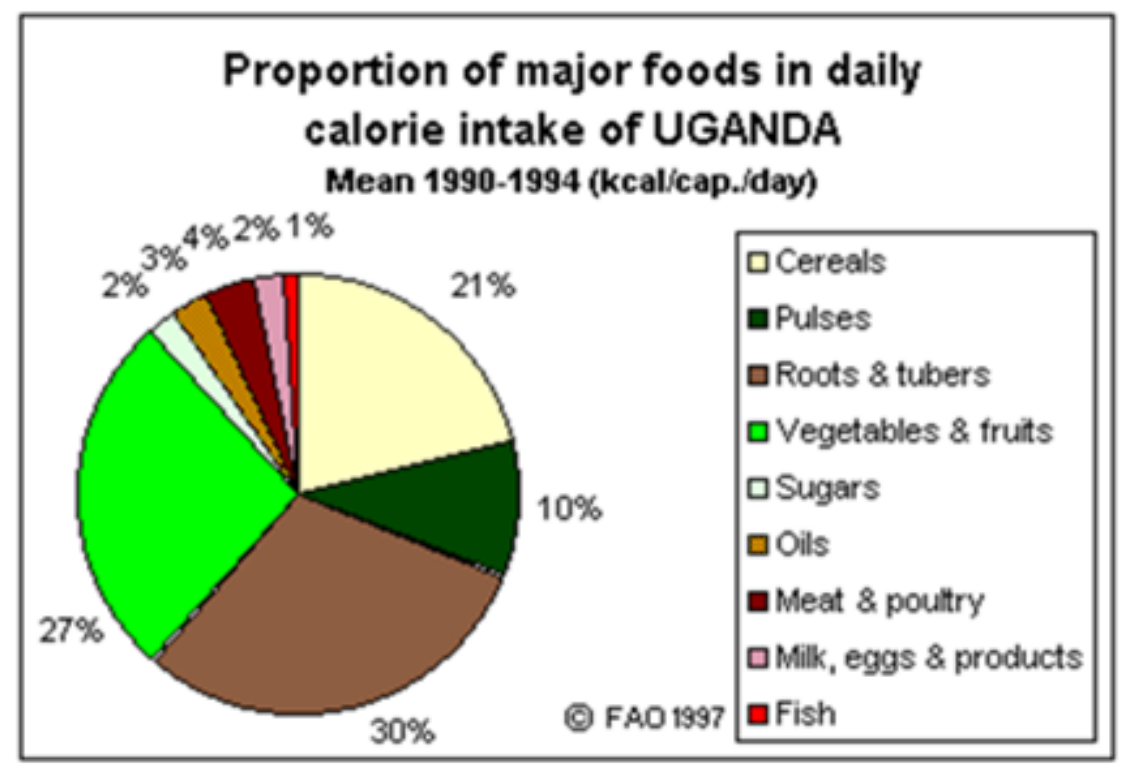


Figure 2: UGANDA: Cereal Human Consumption

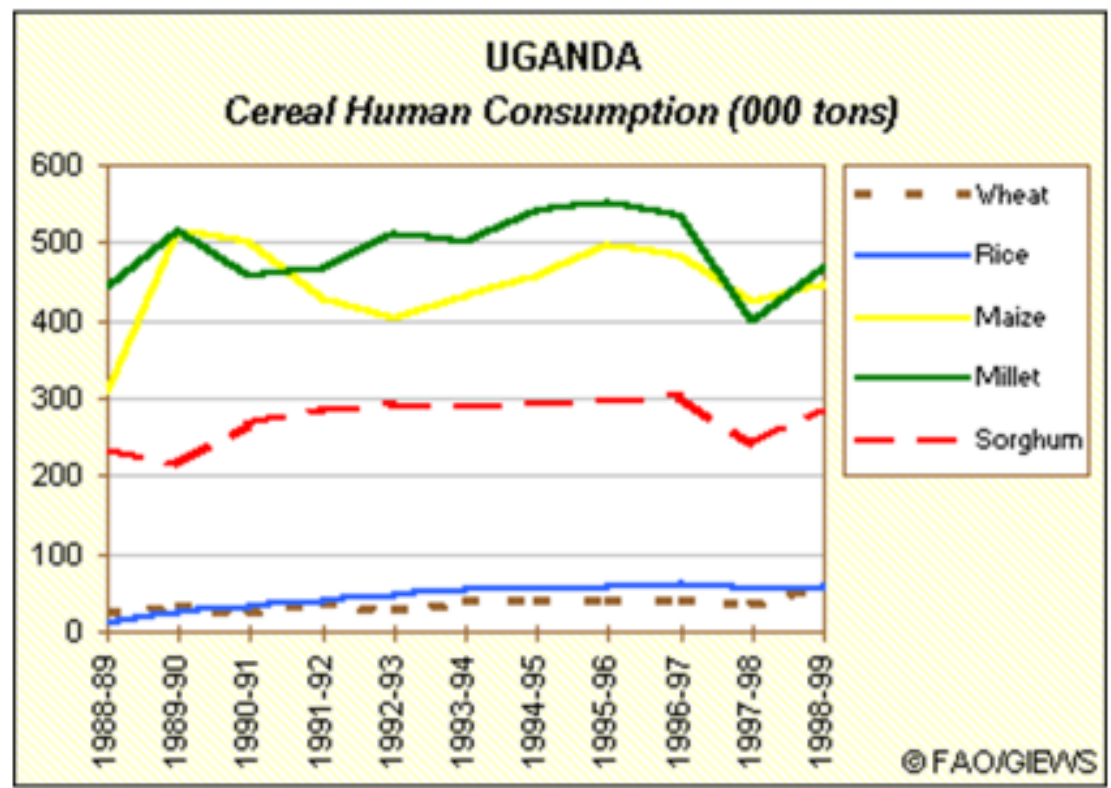

The purpose of this study was to analyze starchy food demand patterns of Ugandan households and conduct econometrics analysis utilizing eleven different variables, namely: income levels, price, regional dummy, urbanization status of the household, production of food by household, border-effect as well as socio-demographic characteristics such as size of household, education status of head of household, sex of head of household, age of head of household.

The main objective was to test the hypothesis as to whether consumers in poorer countries resort to greater substitution within the starchy food groups (i.e. cereals). The Working (1943) model was used to estimate aggregate expenditures and price elasticities for aggregate demand food and non-food commodities while the Linear Approximation of Almost Ideal Demand System (LAAIDS), was tested econometrically for 5 starchy food commodities.

To accomplish this, the 1996/1997 Uganda National Household Budget Survey (UNHS) data from the Uganda Bureau of Statistics (UBOS) are used. In this study the Heckman's two-step model was used to correct for zero consumption.

\section{Literature Review}

Savadogo and Brandt (1988) in Burkina Faso used the LA/AIDS model and showed that two-thirds of the cereal budget was allocated to rice and wheat. Reardon et al. (1992) also utilizing the LA/AIDS model showed that rice is the main urban staple food for both low- and high-income households inBurkina Faso. Nweke et al. (1992), in a study that covered southeastern Nigeria, estimated, using an OLS-Instrumental Variables method, elasticities of demand for major food items in a root- and tuber-based food system. In this study cassava was found to be the next most important staple food, especially for low-income households. Cassava product (gari) is a normal good, and its consumption increases as income increases among high-income urban households. Dorosh et al. (1994) estimated income and price elasticities using survey data collected in the year 1991-1992 from 1816 households in the greater Maputo, Mozambique. The results from this study show that expenditure on food, as a percentage of income, was $80 \%$ for the poor and $65 \%$ for the non-poor.

\section{Model Specification}

This study will apply the LA/AIDS model, which was developed by Deaton and Muellbauer (1980a, 1980b). To begin, an AIDS model for the 14 food commodities is estimated as follows:

$$
w_{i}=\alpha_{t}+\sum_{j} \gamma_{i j} \ln (p j)+\beta_{j} \ln \left(\frac{x}{p}\right)+\mu_{t}, \mathrm{i}=1, \ldots \ldots 14
$$

where $w_{i}(\geq 0)$ is the budget share of food product $i, p_{j}$ is the price of food commodity $j, x$ is the total expenditure on food commodity in question, $\mu_{\mathrm{i}}$ 's are random disturbances assumed with zero mean and constant variance, and $\mathrm{P}$ is a translog price index which is defined by:

$$
\log P=\alpha_{t}+\sum_{k} \alpha_{\kappa} \ln p_{k}+\frac{1}{2} \sum_{k} \sum_{l} \gamma_{\kappa \lambda} \ln p_{k} \ln p_{l}
$$




$$
\mathrm{k}=1, \ldots ., 14 \quad \mathrm{i}=1, \ldots \ldots, 14
$$

The model defined by the Equations (1) to (2) is called the AIDS model. However, the price index in Equation (2) raises difficulties of estimation because of non-linearity in parameters. To avoid the non-linearity problem, Asche and Wessells (1997) suggested the application of the Stone index, which is widely used for LA/AIDS estimation. Moschini (1995) suggested the creation of a log-linear analog of the

Laspeyres price indexes as:

$$
\ln \left(P^{*}\right)=\sum_{j} w_{i} \ln \left(p_{i}\right), \quad \mathrm{i}=1, \ldots \ldots, 14
$$

Where $\mathrm{w}$ is the budget share among 14 commodities. The Stone index is an approximation proportional to the translog, which means that $\mathrm{P}=\varphi \mathrm{P}^{*}$ where $\mathrm{E}(\ln (\varphi))=\alpha_{0}$. The LA/AIDS model with the Stone index is, therefore,

$$
w_{i}=\alpha_{t}^{*}+\sum_{j} \gamma_{i j} \ln \left(p_{j}\right)+\beta_{t} \ln \left(\frac{x}{p^{*}}\right)+\mu_{t}^{*},
$$

Where $\alpha_{i}^{*}=\alpha_{t}-\beta_{i} \alpha_{i}$ and $\mu_{i}^{*}=\mu_{i}-\beta_{i}(\ln (\varphi)-E(\ln (\varphi))$.

According to Moschini (1995), prices will never be perfectly collinear. He found that applying the Stone index will introduce the units of measurement error. To overcome this measurement error problem, Moschini (1995) suggested the log-linear analogue of the Laspeyres price index be obtained by replacing $w_{i}$ in Equation (3) with $\bar{w}_{i}$, which implies mean budget share. The Laspeyres price index, therefore, becomes a geometrically weighted average of prices:

$$
\ln \left(P^{L}\right)=\sum_{i} \bar{w}_{i} \ln \left(P_{i}\right)
$$

When (5) is substituted into (4), it yields an LA/AIDS model with the Laspeyres price index as follows:

$$
w_{i}=\alpha_{i}^{* *}+\sum_{j} \gamma_{i j} \ln \left(p_{j}\right)+\beta_{i}\left(\ln (x)-\sum_{j} \bar{w}_{j} \ln \left(p_{j}\right)\right)+\mu_{i}^{* *}
$$

Where $w_{i}=\alpha_{i}^{* *}=\alpha_{i}-\beta_{i}\left(\alpha_{0}-\sum_{j} \bar{w}_{j} \ln \left(p_{j}\right)\right)$

To conform to microeconomic theory, the adding-up, homogeneity, and symmetry properties of a demand function can be imposed on the LA/AIDS parameters. The adding-up restriction is satisfied with given $\sum_{i} w_{i}=1$ for all $\mathbf{j}$;

$$
\sum_{i} \alpha_{t}=1, \sum_{i} \beta_{\imath}=0, \text { And } \sum_{k} \gamma_{k j}=0
$$

The homogeneity restriction is satisfied for the LA/AIDS model, if for all $\mathrm{j}$,

$$
\sum_{k} \gamma_{j k}=0
$$

Symmetry is satisfied by:

$$
\gamma_{i j}=\gamma_{j i} \square
$$

In this study, weak separability is assumed so as to allow a two-stage budget process. Food demand will be estimated by applying the Working (1993) model in stage one and LA/AIDS in stage two.

To include socio-demographic factors in this study, the basic LA/AIDS model that has been specified must be extended. This is done by following Pollak and Wales $(1978,1981)$ where they modified the original cost function so that the constant term becomes

$$
\alpha=\alpha+\sum_{j=1}^{n} p_{j} d_{j}
$$

Where $d_{j}$ represents household characteristics. This method is known as a linear demographic translation and is used to preserve the linearity of the system. As a result, the derived system of share equations takes the form:

$$
w_{i}=\alpha_{i}^{* * *}+d+\sum_{j} \gamma_{i j} \ln \left(p_{j}\right)+\beta_{i}\left(\ln (x)-\sum_{j} \bar{w}_{j} \ln \left(p_{j}\right)+\mu_{i}^{* * *}\right.
$$


In the diary records method that was used to collect UNHS data, many zero expenditures are reported. The problem of zero expenditure has to be dealt with because if one includes zero observations in an econometric estimation without special treatment, this would lead to biased and inconsistent estimators (Intriligator et al., 1996). To treat the problem, the Generalized Heckman Procedure that was proposed by Heckman (1979) is applied.

\section{Results and Discussion}

The food budget shares in Uganda are similar to other budget surveys done in Africa (Teklu, 1996). Expenditures in the urban and rural areas shows that high-income and low-income households differ widely in the proportion of income they allocate to their food budgets; low-income households spend over $60 \%$ of their income on food, while higherincome households spend slightly less than one-half of their income on food, as is the case for urban areas in Uganda. The highest food expenditure group comprised meat products, $14.1 \%$, followed by fish products at $10.1 \%$. Expenditures on maize constituted $9.2 \%$, while $9.1 \%$ was spent on sugar products, $8.1 \%$ on rice, $7.6 \%$ on other foods, $6.5 \%$ on dairy products, and $5.7 \%$ was spent on matooke and pulses, respectively. Finally, $4.4 \%$ of the expenditure was on cereals, $4.2 \%$ was spent on fats and oils, $4.1 \%$ on fruits and vegetables, and $2.0 \%$ on soft beverages.

Meat, especially bovine meat, is the staple food among the pastoral communities of Northern, Eastern, and Western Uganda. The budget share for fish may reflect both availability and preference. Uganda has an abundance of lakes and rivers. A typical Ugandan diet consists of ugali, a stiff maize porridge. Amongst the starchy cereals, maize is popular in urban areas. Also popular in the Ugandan diet, especially in the Central region, is matooke, with the fourth highest budget share. Matooke is usually eaten with groundnut stew and this may explain the reason why pulses, although highly aggregated, also have high share values. Rice, unlike maize that is grown in many regions of the country, is cultivated in limited areas. Local production of rice is normally unable to meet the domestic demand and thus some rice is imported.

Compared to the Central Region, purchases of matooke are less likely in the Eastern, Northern, and Western Regions (Table 1a). Households in the Central Region have traditionally consumed more matooke than any other region, but this relationship is reversed when maize is the food crop in question. Compared to the Central Region, the Eastern, Northern, and Western Regions show a greater probability for consuming maize. The Eastern Region alone exhibits a lesser likelihood for consumption of rice than does the Central Region, perhaps explained by the availability of the product grown in this region (Table 1a).

Table 1a. Probit Estimates of Parameters for Ugandan Household Food Purchases, 1999/2000.

\begin{tabular}{|c|c|c|c|c|c|c|c|c|c|c|c|c|c|c|}
\hline $\begin{array}{l}\text { Dependent } \\
\text { Variable }\end{array}$ & & & & & & & & & & & & & & tage 2 \\
\hline \multirow{2}{*}{$\begin{array}{l}\text { Decision to } \\
\text { Purchase: }\end{array}$} & & $\begin{array}{r}\text { OOK } \\
\text { E }\end{array}$ & \multicolumn{2}{|r|}{ MAIZE } & \multicolumn{2}{|r|}{ RICE } & \multicolumn{2}{|r|}{ SUGAR } & \multicolumn{2}{|c|}{ CEREAL } & \multicolumn{2}{|r|}{ OILS } & \multicolumn{2}{|c|}{ FRUIT \& VEGE } \\
\hline & & 1 & & 2 & & 3 & & 4 & & 5 & & 6 & & 7 \\
\hline $\begin{array}{l}\text { Explanatory } \\
\text { variables }\end{array}$ & coeff & t-stat & coeff & t-stat & coeff & t-stat & coeff & t-stat & coeff & t-stat & coeff & t-stat & coeff & t-stat \\
\hline Intercept & $0.371^{\mathrm{a}}$ & $\begin{array}{r}3 . \\
63\end{array}$ & $\begin{array}{r}3.275 \\
\mathrm{a}\end{array}$ & 9.17 & $1.684^{\mathrm{a}}$ & $\begin{array}{r}14.4 \\
7\end{array}$ & $1.537^{\mathrm{a}}$ & 9.82 & $-3.653^{\mathrm{b}}$ & -2.04 & $\begin{array}{r}1.68 \\
8^{\text {a }}\end{array}$ & 11.67 & -1.228 & -1.82 \\
\hline EASTERN & $0.500^{\mathrm{a}}$ & $\begin{array}{r}5.8 \\
6 \\
\end{array}$ & $\begin{array}{r}0.024 \\
\mathrm{c}\end{array}$ & 1.85 & $-0.052^{\mathrm{a}}$ & -4.90 & $0.183^{\mathrm{a}}$ & 8.65 & $0.608^{\mathrm{b}}$ & 2.14 & $\begin{array}{r}- \\
0.017\end{array}$ & -1.41 & -0.096 & -2.98 \\
\hline $\begin{array}{l}\text { NORTHER } \\
\mathbf{N}\end{array}$ & $-0.737^{\mathrm{a}}$ & $\begin{array}{r}4.6 \\
7\end{array}$ & $\begin{array}{r}0.891 \\
\mathrm{a}\end{array}$ & 6.53 & $0.214^{\mathrm{a}}$ & 8.72 & $0.402^{\mathrm{a}}$ & 9.99 & $0.603^{b}$ & 2.14 & $\begin{array}{r}0.06 \\
6^{\mathrm{a}}\end{array}$ & 3.95 & -0.155 & -3.21 \\
\hline WESTERN & $0.079^{\mathrm{a}}$ & $\begin{array}{r}4.5 \\
3 \\
\end{array}$ & $\begin{array}{r}0.102 \\
\mathrm{a}\end{array}$ & 5.01 & $0.042^{\mathrm{a}}$ & 4.14 & $0.256^{\mathrm{a}}$ & 9.32 & $0.317^{\mathrm{b}}$ & 2.11 & $\begin{array}{r}0.23 \\
6^{\mathrm{a}} \\
\end{array}$ & 8.58 & $-0.177^{\mathrm{a}}$ & -3.22 \\
\hline BORDER & $0.265^{\mathrm{a}}$ & $\begin{array}{r}5 . \\
49\end{array}$ & $-0.795^{\mathrm{a}}$ & -6.31 & 0.006 & 0.64 & $-0.120^{a}$ & -7.20 & $0.076^{\mathrm{b}}$ & 2.16 & $0.041_{\mathrm{a}}^{-}$ & -3.06 & $0.236^{\mathrm{a}}$ & 3.18 \\
\hline PCFEXP & $0.008^{\mathrm{a}}$ & $\begin{array}{r}4 . \\
91 \\
\end{array}$ & $0.024^{\mathrm{a}}$ & 6.03 & $0.013^{\mathrm{a}}$ & 5.80 & $0.015^{\mathrm{a}}$ & 7.79 & $0.011^{\mathrm{b}}$ & 2.20 & $\begin{array}{r}0.015 \\
\mathrm{a}\end{array}$ & 7.03 & $0.003^{\mathrm{a}}$ & 2.48 \\
\hline PROD & $0.156^{\mathrm{a}}$ & $\begin{array}{r}4 . \\
95 \\
\end{array}$ & 0.010 & 0.81 & $0.116^{\mathrm{a}}$ & 7.33 & $-0.080^{\mathrm{a}}$ & -5.67 & $0.521^{\mathrm{b}}$ & 2.13 & $\begin{array}{r}0.079 \\
\mathrm{a}\end{array}$ & -5.24 & $1.008^{\mathrm{a}}$ & 2.99 \\
\hline URBAN & $0.117^{\mathrm{a}}$ & $\begin{array}{r}4 . \\
65\end{array}$ & $-0.259^{\mathrm{a}}$ & -6.07 & $-0.278^{\mathrm{a}}$ & -7.80 & $-0.189^{a}$ & -8.56 & $0.030^{\mathrm{b}}$ & 2.10 & 0.201 & -7.71 & $-0.241^{\mathrm{a}}$ & -2.87 \\
\hline
\end{tabular}

Notes: Superscripts a, b and c indicate statistical significance at 99, 95 and 90 percent levels, respectively. Data source: UNHS 1999/2000 
Households located in border districts demonstrate a greater likelihood of purchasing maize, matooke, and meat (Tables 1a, 1b). Border district households also more likely consume more sugar, oils, fruits and vegetables, dairy products, and beverages than do households in the interior districts, while the quantities of staples consumed, such as maize, cereal, and rice, are less likely consumed. Households located in the border districts also exhibit greater probabilities of purchasing alcohol, rice, cereal, matooke, meat, and fish.

Table 1b. Probit Estimates of Parameters for Ugandan Household Food Purchases, 1999/2000.

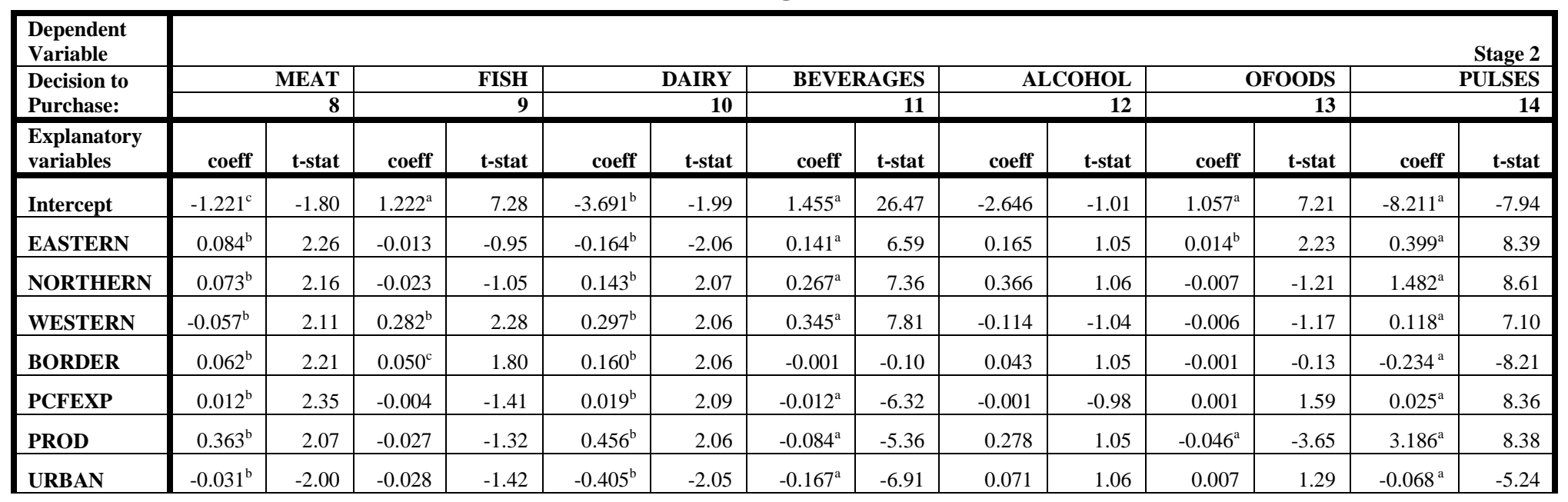

Notes: Superscripts a, b and c indicate statistical significance at 99, 95 and 90 percent levels, respectively. Data source: UNHS 1999/2000

Households that produce some of the food they consume, as expected, have a negative influence on the probability of purchase of matooke, maize, rice, dairy products and a positive influence on food groups that they may be unable to produce on their own - sugar, oils, fruits and vegetables, meat, fish, beverages, alcohol, pulses, and other foods. People living in urban areas consume more matooke, sugar, other cereals, oils, fruits and vegetables, fish, dairy products, other foods, and pulses than their counterparts in the rural areas. The positive cereal coefficient may be an indication that the status of bread consumption outweighs the effect of consuming sorghum and millet and thus supports prior findings. Contrary to other studies' inferences, the consumption of rice for Ugandan households residing in urban settings suggests a negative correlation.

The expenditure coefficients for maize, fat and oil, dairy, and pulses are negative in the LA/AIDS estimations, implying that these food categories are necessities (Tables $2 a, 2 b$ ). On the other hand, the expenditure coefficients for sugar, fish, cereal, fruit and vegetables, meat, and alcohol are positive, which implies that these foods are luxuries. Results further showed that Ugandans with higher incomes consume more rice, fruits and vegetables, and soft beverages than their low-income counterparts. Low-income households consumed more staple food products, such as matooke, maize, and cereals. 
Table 2a. Estimated Parameters of Heckman Two-Stage LA/AIDS model (UNHS 1999/2000).

In this study (Tables $2 a, 2 b$ ), there was a positive and significant correlation between households located in urban areas

\begin{tabular}{|c|c|c|c|c|c|c|c|c|c|c|c|c|c|c|c|c|}
\hline \multirow{3}{*}{$\begin{array}{l}\text { variable } \\
\text { Budget share } \\
\text { variable }\end{array}$} & \multicolumn{4}{|c|}{ Stage 1} & & \multirow{2}{*}{\multicolumn{2}{|c|}{ Maize }} & \multirow{2}{*}{\multicolumn{2}{|c|}{ Rice }} & \multirow{2}{*}{\multicolumn{2}{|c|}{ Sugar }} & & & & & tage 2 \\
\hline & FOOD & & N- FOOD & & Matooke & & & & & & & \multicolumn{2}{|c|}{ Cereal } & \multicolumn{3}{|c|}{ Fats \& Oils } \\
\hline & coeff & t-stat & coeff & t-stat & coeff & t-stat & coeff & t-stat & coeff & t-stat & coeff & t-stat & coeff & t-stat & coeff & t-stat \\
\hline Intercept & $0.406^{\mathrm{a}}$ & 6.50 & $0.585^{\mathrm{a}}$ & 8.68 & -0.008 & -0.09 & $-0.083^{c}$ & -1.86 & $0.094^{\circ}$ & 1.91 & 0.032 & 0.74 & $-0.392^{a}$ & -2.59 & 0.005 & 0.18 \\
\hline CENTRAL & $-0.021^{\mathrm{a}}$ & -7.64 & $0.024^{\mathrm{a}}$ & 8.48 & 0.020 & 0.44 & -0.029 & -1.22 & 0.018 & 0.70 & -0.025 & -1.03 & 0.009 & 0.20 & 0.014 & 0.90 \\
\hline EASTERN & $0.009^{\mathrm{a}}$ & 3.16 & -0.003 & -0.94 & -0.049 & -0.94 & $-0.039^{c}$ & -1.67 & -0.005 & -0.19 & $-0.050^{\mathrm{b}}$ & -2.14 & 0.059 & 1.20 & -0.003 & -0.18 \\
\hline WESTERN & $-0.012^{\mathrm{a}}$ & -4.22 & $0.016^{\mathrm{a}}$ & 5.18 & -0.019 & -0.43 & 0.011 & 0.43 & 0.010 & 0.36 & $-0.060^{\mathrm{b}}$ & -2.49 & 0.068 & 1.44 & 0.025 & 1.57 \\
\hline BORDER & 0.002 & 0.81 & -0.002 & -0.67 & $0.092^{b}$ & 2.42 & $0.035^{c}$ & 1.88 & 0.001 & 0.03 & $0.042^{\mathrm{b}}$ & 2.23 & $0.062^{c}$ & 1.81 & $0.032^{\mathrm{a}}$ & 2.61 \\
\hline HHSIZE & 0.001 & 1.32 & -0.001 & -1.10 & $-0.021^{b}$ & 2.09 & -0.007 & -1.19 & -0.008 & -1.34 & 0.002 & 0.38 & -0.015 & -1.52 & -0.005 & -1.37 \\
\hline HHAGE & -0.000 & -0.98 & 0.000 & 0.89 & $0.001^{\mathrm{b}}$ & 1.96 & -0.000 & -0.05 & 0.000 & 0.24 & 0.000 & 0.87 & -0.000 & 0.00 & 0.000 & 0.06 \\
\hline HHFEM & $0.005^{\circ}$ & 1.89 & $-0.005^{\mathrm{c}}$ & -1.83 & $-0.045^{\mathrm{c}}$ & -1.70 & 0.007 & 0.49 & 0.017 & 1.06 & 0.000 & 0.01 & -0.027 & -0.99 & 0.009 & 0.99 \\
\hline HHHMS & $-0.002^{\mathrm{b}}$ & -1.97 & $0.002^{\mathrm{b}}$ & 2.08 & -0.004 & -0.24 & 0.009 & 1.13 & $-0.014^{\circ}$ & -1.70 & 0.004 & 0.49 & -0.008 & -0.53 & -0.006 & -1.22 \\
\hline N1 $(<6)$ & $-0.004^{\mathrm{a}}$ & -2.65 & $0.004^{a}$ & 2.58 & $0.024^{c}$ & 1.70 & 0.004 & 0.55 & 0.012 & 1.42 & 0.001 & 0.12 & 0.012 & 0.88 & $0.008^{\mathrm{c}}$ & 1.67 \\
\hline N2 ( 7 to 12) & -0.002 & -1.53 & 0.002 & 1.23 & 0.019 & 1.16 & 0.012 & 1.35 & 0.004 & 0.38 & -0.000 & -0.04 & 0.017 & 1.04 & 0.003 & 0.21 \\
\hline N3 (13 to 19) & -0.002 & -1.25 & 0.002 & 1.13 & $0.047^{\mathrm{a}}$ & 3.02 & 0.008 & 0.96 & 0.010 & 1.08 & -0.000 & -0.06 & 0.017 & 1.09 & 0.005 & 0.82 \\
\hline N4 (20 to 54$)$ & -0.001 & -0.53 & 0.001 & 0.52 & $0.038^{\mathrm{b}}$ & 2.08 & 0.013 & 1.40 & 0.000 & 0.02 & -0.002 & -0.21 & 0.029 & 1.61 & $0.013^{\mathrm{c}}$ & 1.95 \\
\hline PROD & $-0.039^{\mathrm{a}}$ & -7.26 & 0.044 & 1.34 & $-0.046^{\circ}$ & -1.70 & -0.003 & -0.22 & $0.040^{b}$ & 2.33 & $0.027^{\mathrm{c}}$ & 1.81 & 0.046 & 1.47 & -0.011 & -1.09 \\
\hline TCEXP1 & $-0.001^{\mathrm{a}}$ & -7.30 & $0.001^{\mathrm{a}}$ & 7.34 & 0.001 & 0.54 & $0.002^{\mathrm{c}}$ & 1.65 & $0.002^{\mathrm{c}}$ & 1.89 & 0.000 & 0.22 & 0.001 & 0.26 & 0.000 & 0.27 \\
\hline TCEXP2 & $0.000^{\mathrm{a}}$ & 5.68 & $-0.000^{\mathrm{a}}$ & -5.62 & $-0.000^{\mathrm{a}}$ & -2.85 & $-0.000^{c}$ & -1.63 & 0.000 & 0.05 & -0.000 & -1.21 & -0.000 & -0.54 & -0.017 & -0.62 \\
\hline URBAN & $0.004^{\circ}$ & 1.67 & -0.004 & -1.52 & 0.001 & 0.05 & 0.012 & 1.17 & -0.006 & -0.58 & 0.004 & 0.37 & 0.010 & 0.53 & 0.006 & 0.89 \\
\hline QUARTER & -0.001 & -0.30 & 0.001 & 0.41 & -0.035 & -0.98 & -0.026 & -1.41 & 0.003 & 0.15 & $0.011^{\mathrm{c}}$ & 1.79 & 0.015 & 0.43 & -0.006 & -0.48 \\
\hline HHEducation & $0.004^{b}$ & 1.94 & $-0.005^{\mathrm{b}}$ & -2.11 & $-0.020^{c}$ & -1.63 & $0.014^{\mathrm{b}}$ & 2.12 & $-0.012^{c}$ & -1.75 & -0.010 & -1.49 & -0.017 & -1.42 & -0.003 & -0.66 \\
\hline ITCEXPp & $-0.055^{\mathrm{a}}$ & -6.86 & $0.055^{\mathrm{a}}$ & 4.90 & 0.010 & 0.78 & $-0.014^{b}$ & -1.98 & -0.006 & -0.74 & 0.001 & 0.15 & 0.010 & 0.79 & -0.001 & -0.15 \\
\hline lpmatook & & & & & $0.060^{b}$ & 2.43 & -0.000 & -0.04 & -0.001 & -0.05 & -0.000 & -0.08 & 0.013 & 0.53 & $0.023^{\mathrm{a}}$ & 2.65 \\
\hline lpmaize & & & & & 0.068 & 1.27 & 0.026 & 0.89 & 0.030 & 0.96 & 0.040 & 1.38 & 0.010 & 0.20 & 0.032 & 1.68 \\
\hline lprice & & & & & -0.062 & -1.03 & 0.018 & 0.58 & -0.008 & -0.23 & -0.005 & -0.16 & 0.061 & 1.05 & -0.022 & -1.00 \\
\hline Ipsugar & & & & & $-0.071^{\mathrm{b}}$ & -2.04 & 0.004 & 0.21 & 0.003 & 0.14 & -0.006 & -0.30 & -0.017 & -0.51 & 0.003 & 0.25 \\
\hline lpcereal & & & & & $0.179^{b}$ & 2.51 & -0.050 & -1.32 & 0.014 & 0.34 & -0.021 & -0.55 & 0.015 & 0.21 & 0.005 & 0.18 \\
\hline Ipfoil & & & & & -0.004 & -0.31 & -0.005 & -0.71 & 0.006 & 0.83 & $-0.019^{\mathrm{a}}$ & -2.77 & -0.007 & -0.56 & -0.002 & -0.45 \\
\hline lpfeg & & & & & 0.006 & 0.26 & 0.008 & 0.67 & -0.013 & -1.06 & 0.013 & 1.17 & -0.001 & -0.05 & 0.008 & 1.04 \\
\hline lpmeat & & & & & $-0.298^{\mathrm{a}}$ & -2.82 & -0.048 & -0.85 & $-0.147^{b}$ & -2.36 & $-0.098^{\mathrm{c}}$ & -1.74 & -0.099 & -0.95 & -0.042 & -1.12 \\
\hline lpfish & & & & & -0.016 & -1.16 & 0.000 & 0.01 & 0.001 & 0.07 & -0.002 & -0.27 & 0.005 & 0.36 & 0.001 & 0.22 \\
\hline lpdairy & & & & & $0.552^{b}$ & 2.06 & 0.098 & 0.72 & $0.384^{b}$ & 2.56 & 0.017 & 0.12 & $0.409^{c}$ & 1.66 & $0.213^{\mathrm{b}}$ & 2.41 \\
\hline lpbev & & & & & 0.002 & 0.24 & -0.001 & -0.45 & 0.003 & 0.84 & -0.005 & -1.47 & -0.001 & -0.15 & -0.000 & -0.11 \\
\hline Ipalcohol & & & & & 0.047 & 0.84 & -0.037 & -1.23 & 0.007 & 0.21 & -0.037 & -1.27 & 0.043 & 0.79 & $0.066^{\mathrm{a}}$ & 3.39 \\
\hline lppulses & & & & & -0.001 & -0.02 & -0.008 & -0.42 & $-0.042^{\mathrm{b}}$ & -2.04 & -0.004 & -0.20 & 0.033 & 0.98 & -0.017 & -1.41 \\
\hline MR & $-0.061^{\mathrm{a}}$ & -5.73 & $0.950^{\mathrm{a}}$ & 7.45 & -0.008 & -0.19 & 0.007 & 0.35 & 0.004 & 0.32 & -0.006 & -0.31 & $0.132^{\mathrm{a}}$ & 2.78 & $0.024^{\mathrm{b}}$ & 2.25 \\
\hline
\end{tabular}

and the consumption of fruit and vegetables. There was also a strong and positive correlation between these households and the consumption of matooke, maize, sugar, cereal, fats and oil, fish, dairy products, and alcohol. Households that reside in the border districts of Uganda consume significantly higher amounts of matooke, sugar, oils, fruits and vegetables, dairy products, alcohol and pulses than do households in the interior districts.

Table 2b. Estimated Parameters of Heckman Two-Stage LA/AIDS model (UNHS data, 1999/2000)

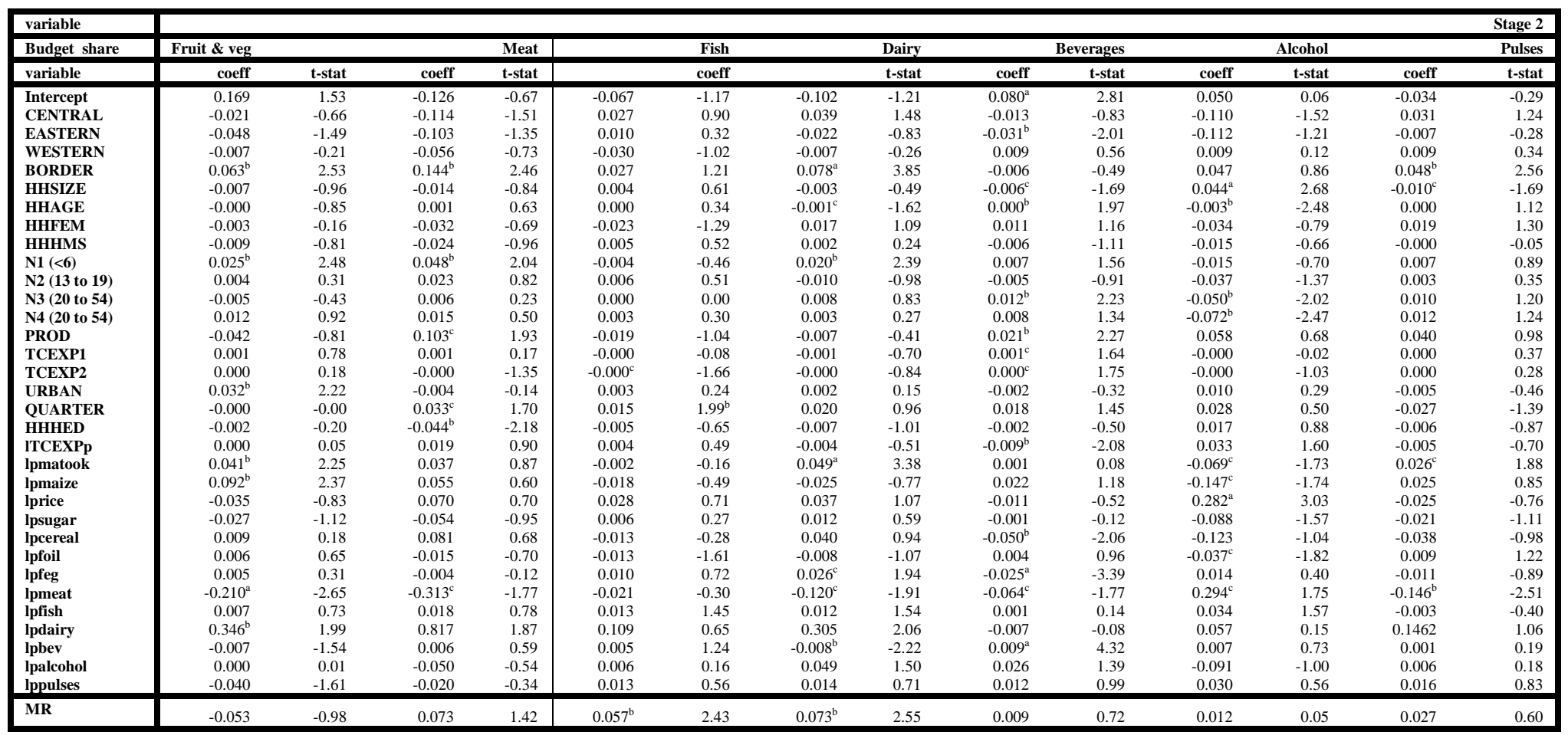

Notes: Superscripts a, b and c indicate statistical significance at 99, 95 and 90 percent confidence levels, respectively.

Education attainment of the head of household had a positive, significant correlation with food consumption. When the individual food groups are scrutinized (Tables $2 a, 2 b$ ), households with heads that possess a higher education consume significantly higher amounts of maize and alcohol. Female headed households consumed more maize, rice, dairy 
products, sugar, beverages, and pulses, but less matooke, cereals, fats and oils, fish, and meats than male-headed households. There was also a positive and significant correlation between households with children under the age 6 (N1) and the consumption of food products, such as dairy products, meat, matooke, fats and oil, and fruits and vegetables. Households with members aged 13 to 19 (N3) and aged 20 to 55 (N4) consumed significantly larger amounts of matooke and fats and oils than their counterparts aged over 55 (N5) and the consumption of maize, cereal, rice, and beverage is important to households with these age groups.

Households that engaged in production of matooke experienced significant, reduced consumption shares of this food product relative to households that were not engaged in matooke production. Seasonal coefficients had significant explanatory influence in the consumption of meat, fish, and sugar. The expenditure elasticities (Tables 3a, 3b), for food and for all food groups are positive, implying that food is a normal good. The point elasticity estimates for matooke, maize, cereal, fish, meat, and pulses are greater than unity, implying that for these food categories, an increase in total food expenditures will result in more than proportionate increase in expenditure shares. On the other hand, estimates for rice, sugar, fruit and vegetables, meat, dairy products, and soft beverages are all less than unity, implying that an increase in future expenditures on food will result in less than proportionate increases in expenditures on these food groups.

Table 3a: Uncompensated Price and Expenditure Elasticities: LA/AIDS with Inverse Mills Ratio

\begin{tabular}{|c|c|c|c|c|c|c|c|c|c|c|c|c|c|c|c|}
\hline Food Item & & & & & & & & & & & & & & Uncompen & ed price elasticity \\
\hline & $\begin{array}{l}\text { Mean budget } \\
\text { share }\end{array}$ & mat & maize & rice & sugar & cereal & oil & veg & meat & fish & dairy & bev & alcoh & pulses & $\begin{array}{l}\text { EXPEND. } \\
\text { Elasticity }\end{array}$ \\
\hline Food & $44 \%$ & & & & & & & & & & & & & & 1.48261 \\
\hline Non-food & $56 \%$ & & & & & & & & & & & & & & 1.84623 \\
\hline matooke & $5.7 \%$ & -0.622 & 0.078 & -0.197 & -0.089 & -0.168 & 0.315 & 0.459 & 0.069 & -0.098 & 0.183 & 0.062 & -0.547 & 0.212 & 1.04914 \\
\hline maize & $9.2 \%$ & 0.130 & -0.417 & -0.397 & 0.148 & 0.740 & 1.005 & 1.829 & -0.249 & 0.014 & 0.180 & -0.555 & -1.999 & 0.726 & 1.09070 \\
\hline rice & $8.1 \%$ & -0.278 & -0.358 & -0.435 & -0.105 & 0.229 & -0.930 & -0.765 & 0.399 & -0.035 & 0.390 & 0.731 & 2.493 & -4.846 & 0.89568 \\
\hline sugar & $9.1 \%$ & -0.134 & 0.144 & -0.113 & -0.701 & 0.220 & 0.211 & 0.033 & -0.038 & 0.255 & 0.499 & 0.588 & -0.825 & -0.470 & 1.00623 \\
\hline cereal & $4.4 \%$ & -0.120 & 0.378 & 0.139 & 0.116 & -0.980 & 0.070 & -0.107 & 0.181 & -0.196 & 0.759 & -1.716 & -0.995 & 0.867 & 1.12345 \\
\hline oil & $4.2 \%$ & 0.213 & 0.461 & -0.481 & 0.096 & 0.059 & -1.022 & 0.166 & 0.021 & -0.104 & -0.040 & 0.187 & -0.257 & -0.010 & 0.96308 \\
\hline veg & $4.1 \%$ & 0.306 & 0.846 & -0.399 & 0.012 & -0.109 & 0.167 & -1.290 & -0.095 & 0.022 & -0.160 & -0.594 & 0.054 & -0.448 & 0.85339 \\
\hline meat & $14.1 \%$ & 0.135 & -0.417 & 0.730 & -0.068 & 0.532 & 0.073 & -0.315 & -0.968 & 0.341 & -0.636 & -0.788 & 3.064 & -1.886 & 0.91728 \\
\hline fish & $10.2 \%$ & -0.171 & 0.015 & -0.043 & 0.306 & -0.459 & -0.268 & 0.064 & 0.261 & -0.857 & 0.196 & 0.530 & 0.396 & -1.822 & 1.02392 \\
\hline dairy & $6.5 \%$ & 0.199 & 0.128 & 0.315 & 0.358 & 1.055 & -0.060 & -0.240 & -0.280 & 0.117 & -1.045 & -1.715 & -1.750 & 1.077 & 1.04017 \\
\hline beverage & $2.0 \%$ & 0.003 & -0.134 & 0.189 & 0.131 & -0.787 & 0.090 & -0.304 & -0.124 & 0.099 & -0.561 & -0.488 & 0.065 & 1.003 & 0.55063 \\
\hline alcohol & $9.6 \%$ & -0.906 & -1.239 & 1.147 & -0.921 & -2.172 & -0.617 & 0.151 & 2.158 & 0.370 & -1.744 & 0.364 & -1.272 & 1.488 & 1.05323 \\
\hline pulses & $5.6 \%$ & 0.197 & 0.423 & -1.350 & -0.290 & 1.015 & 0.003 & -0.536 & -1.251 & -0.953 & 1.539 & 2.845 & 0.818 & -1.440 & 1.16824 \\
\hline
\end{tabular}

Data source: UNHS 1999/2000

Table 3b: Compensated Price and Expenditure Elasticities: LA/AIDS with Inverse Mills Ratio

\begin{tabular}{|c|c|c|c|c|c|c|c|c|c|c|c|c|c|c|c|}
\hline Food Item & & & & & & & & & & & & & & ompensat & price elasticity \\
\hline & $\begin{array}{l}\text { Mean } \\
\text { budget } \\
\text { share }\end{array}$ & mat & maize & rice & sugar & cereal & oil & veg & meat & fish & dairy & bev & alcoh & pulses & $\begin{array}{l}\text { EXPEND } \\
\text { Elasticity }\end{array}$ \\
\hline Food & $44 \%$ & & & & & & & & & & & & & & 4826 \\
\hline Non Food & $56 \%$ & & & & & & & & & & & & & & 84623 \\
\hline matooke & $5.7 \%$ & -0.562 & 0.140 & -0.145 & -0.032 & -0.104 & 0.370 & 0.508 & 0.122 & -0.039 & 0.242 & 0.093 & -0.486 & 0.279 & 1.04914 \\
\hline maize & $9.2 \%$ & 0.219 & -0.324 & -0.320 & 0.234 & 0.836 & 1.087 & 1.902 & -0.170 & 0.102 & 0.269 & -0.508 & -1.909 & 0.826 & 1.09070 \\
\hline rice & $8.1 \%$ & -0.198 & -0.275 & -0.367 & -0.028 & 0.314 & -0.857 & -0.701 & 0.468 & 0.042 & 0.469 & 0.772 & 2.573 & -4.757 & 0.89568 \\
\hline sugar & $9.1 \%$ & -0.045 & 0.237 & -0.036 & -0.615 & 0.316 & 0.293 & 0.106 & 0.039 & 0.342 & 0.588 & 0.635 & -0.735 & -0.371 & 1.00623 \\
\hline cereal & $4.4 \%$ & -0.074 & 0.425 & 0.178 & 0.160 & -0.830 & 0.113 & -0.070 & 0.221 & -0.151 & 0.804 & -1.692 & -0.948 & 0.918 & 1.12345 \\
\hline oil & $4.2 \%$ & 0.254 & 0.503 & -0.446 & 0.136 & 0.103 & -0.984 & 0.201 & 0.057 & -0.064 & 0.001 & 0.208 & -0.215 & 0.035 & 0.96308 \\
\hline veg & $4.1 \%$ & 0.347 & 0.890 & -0.363 & 0.052 & -0.064 & 0.205 & -1.256 & -0.059 & 0.063 & -0.119 & -0.572 & 0.096 & -0.401 & 0.85339 \\
\hline meat & $14.1 \%$ & 0.278 & -0.267 & 0.852 & 0.068 & 0.686 & 0.204 & -0.198 & -0.843 & 0.481 & -0.495 & -0.713 & 1.208 & -1.726 & 0.91728 \\
\hline fish & $10.2 \%$ & -0.064 & 0.127 & 0.047 & 0.409 & -0.344 & -0.171 & 0.151 & 0.354 & -0.753 & 0.303 & 0.586 & 0.504 & -1.702 & 1.02392 \\
\hline dairy & $6.5 \%$ & 0.263 & 0.195 & 0.370 & 0.419 & 1.123 & -0.002 & -0.188 & -0.224 & 0.180 & -1.028 & -1.681 & -1.686 & 1.148 & 1.04017 \\
\hline beverage & $2.0 \%$ & 0.024 & -0.112 & 0.207 & 0.151 & -0.764 & 0.109 & -0.287 & -0.105 & 0.120 & -0.540 & -0.477 & 0.086 & 1.026 & 0.55063 \\
\hline alcohol & $9.6 \%$ & -0.806 & -1.135 & 1.233 & -0.825 & -1.065 & -0.525 & 0.233 & 1.245 & 0.468 & -2.644 & 0.416 & -1.072 & 1.600 & 1.05323 \\
\hline pulses & $5.6 \%$ & 0.253 & 0.481 & $-1,303$ & -0.237 & 1.075 & 0.054 & -0.490 & -1203 & -0.898 & 1595 & 1874 & 0.874 & -1.202 & 1.16824 \\
\hline
\end{tabular}

Data source: UNHS 1999/2000 
Own-price elasticities for all food groups carried the expected negative sign. Own-price elasticities for alcohol, pulses, dairy, fruits and vegetables, and fats and oil are elastic, while staple food products, such as matooke, maize, rice, sugar, and cereals, are inelastic to price changes. Ugandan consumers consider pulses, dairy, meat, oils, sugar, rice, and maize as substitutes for cereals. However, cereal demand complements fruits and vegetables, soft beverages, and alcohol. Ugandan consumers view vegetables as a complement of rice, cereals, meat, dairy, beverages, and pulses, while pulses complement meat, vegetables, and fish consumption.

The results of the estimates for the compensated cross-price elasticities (Table 3b) indicates that these elasticities are fairly low, as compared to the uncompensated elasticities in almost all categories, but they do indicate that some food categories aregross complements, while others are gross substitutes. The cross-price elasticities ofcereal demand with respect to the price for pulses, dairy, meat, oils, sugar, rice, and maize have positive signs, which imply that consumers view these products as substitutes. This result implies that an increase in the price of cereal will lead to Ugandan consumers increasing their demand for maize, rice, sugar, oils, meat, dairy, and pulses. This further indicates that a $10 \%$ increase in the price of cereal will lead to an $8.4 \%, 3.1 \%, 3.1 \%, 1 \%, 6.8 \%, 11.2 \%$, and $10.7 \%$ increase in the demand of maize, rice, sugar, oils, meat, dairy, and pulses, respectively. The cross-price elasticities of cereal demand with respect to the prices of fruits and vegetables, beverages, and alcohol are negative, which implies that these food products are complements. Vegetables have negative signs vis-à-vis rice, cereal, meat, dairy, beverages, and pulses . This leads to the conclusion that Ugandan consumers view vegetables as a complement of rice, cereal, meat, dairy, beverages, and pulses. Another important result is that pulses have a negative relationship with the consumption of meat, vegetables, and fish, indicating that pulses are considered complements of meat, vegetables, and fish.

Calculated price and expenditure elasticities for low-income households are shown on tables $4 \mathrm{a}, 4 \mathrm{~b}$. In these tables, only the cross-price elasticities are considered for discussion. Compensated cross-price elasticities for cereal demand with respect to the prices of matooke, maize, rice, sugar, dairy products, and pulses are all positive, implying that these foods are substitutes. This result indicates that at lower incomes, price changes results in greater consumer substitution within the starchy food groups. At the mean expenditures, the substitution within the starchy foods only occurs within maize, rice, sugar, and pulses. However, at the lower expenditures, this occurs within the starchy food groups of matooke, maize, rice, sugar, and pulses.

\section{Table 4a: Uncompensated Price and Expenditure Elasticities: LA/AIDS with Inverse Mills Ratio (LOW INCOME)}

\begin{tabular}{|c|c|c|c|c|c|c|c|c|c|c|c|c|c|c|c|}
\hline \multirow[t]{2}{*}{ Food Item } & \multirow[b]{2}{*}{$\begin{array}{l}\text { Mean } \\
\text { budget } \\
\text { share }\end{array}$} & \multirow[b]{2}{*}{ mat } & \multirow[b]{2}{*}{ maize } & \multirow[b]{2}{*}{ rice } & \multirow[b]{2}{*}{ sugar } & \multirow[b]{2}{*}{ cereal } & \multirow[b]{2}{*}{ oil } & \multirow[b]{2}{*}{ veg } & \multirow[b]{2}{*}{ meat } & \multirow[b]{2}{*}{ fish } & \multirow[b]{2}{*}{ dairy } & \multirow[b]{2}{*}{ bev } & \multicolumn{3}{|c|}{ Uncompensated price elasticity } \\
\hline & & & & & & & & & & & & & alcoh & pulses & $\begin{array}{l}\text { EXPEND. } \\
\text { Elasticity }\end{array}$ \\
\hline Food & $44 \%$ & & & & & & & & & & & & & & 1.48261 \\
\hline Non-food & $56 \%$ & & & & & & & & & & & & & & 1.84623 \\
\hline matooke & $5.7 \%$ & -1.110 & 0.174 & 0.046 & 0.003 & 0.015 & 0.331 & 0.419 & 0.0182 & 0.041 & 0.303 & 0.025 & 0.021 & -1.328 & 1.38963 \\
\hline maize & $9.2 \%$ & 0.270 & -0.778 & -0.130 & -0.169 & 0.810 & 0.332 & 0.676 & -0.395 & -0.170 & -0.032 & -0.946 & -0.539 & 1.089 & 1.06968 \\
\hline rice & $8.1 \%$ & -0.012 & -0.121 & -0.638 & 0.086 & 0.456 & -0.312 & 0.228 & 0.563 & 0.085 & 0.453 & 0.5653 & 0.822 & -1.587 & 0.67178 \\
\hline sugar & $9.1 \%$ & -0.034 & -0.174 & 0.115 & -0.975 & 0.629 & -0.153 & -0.778 & 4.839 & 1.083 & 2.202 & 2.097 & -1.881 & -1.290 & 0.93375 \\
\hline cereal & $4.4 \%$ & 0.008 & 0.413 & 0.279 & 0.330 & -1.404 & -0.053 & -0.962 & -0.708 & -0.224 & 0.513 & -0.840 & -0.059 & 1.399 & 1.11284 \\
\hline oil & $4.2 \%$ & 0.221 & 0.152 & -0.160 & -0.071 & -0.050 & -1.126 & -0.349 & 0.147 & -0.126 & -0.089 & 0.108 & -0.079 & 0.325 & 0.95997 \\
\hline veg & $4.1 \%$ & 0.258 & 0.310 & 0.137 & -0.363 & -0.8852 & -0.352 & -0.616 & -0.148 & 0.171 & 0.211 & -0.845 & -0.061 & 0.548 & 0.89534 \\
\hline meat & $14.1 \%$ & 0.078 & -0.633 & 1.051 & 7.769 & -2.185 & 0.520 & -0.460 & -1.752 & 0.279 & -0.498 & -1.330 & 1.483 & -1.480 & 1.19698 \\
\hline fish & $10.2 \%$ & 0.077 & -0.203 & 0.117 & 1.299 & -0.519 & -0.325 & 0.444 & 0.209 & -0.594 & 0.172 & 0.989 & 0.717 & -1.848 & 1.02799 \\
\hline dairy & $6.5 \%$ & 0.342 & -0.017 & 0.326 & 1.561 & 0.717 & -0.144 & 0.301 & -0.207 & 0.105 & -0.155 & -4.845 & -1.126 & -1.187 & 0.86276 \\
\hline beverage & $2.0 \%$ & -0.063 & -0.227 & 0.166 & 0.480 & -0.405 & 0.054 & -0.438 & -0.219 & 0.189 & -1.538 & -0.444 & 0.122 & 1.586 & 0.14338 \\
\hline pulses & $5.6 \%$ & -1.231 & 0.6704 & -1.155 & -1.741 & 1.909 & 0.448 & 0.776 & -1.481 & -1.524 & -1.051 & 1.443 & 0.123 & -1.385 & 1.40059 \\
\hline
\end{tabular}

Data source: UNHS 1999/2000 
Table 4b: Compensated Price and Expenditure Elasticities: LA/AIDS with Inverse Mills Ratio (LOW INCOME)

\begin{tabular}{|c|c|c|c|c|c|c|c|c|c|c|c|c|c|c|c|}
\hline Food Item & & & & & & & & & & & & & \multicolumn{3}{|c|}{ compensated price elasticity } \\
\hline & $\begin{array}{l}\text { Mean } \\
\text { budget } \\
\text { share }\end{array}$ & mat & maize & rice & sugar & cereal & oil & veg & meat & fish & dairy & bev & alcoh & pulses & $\begin{array}{l}\text { EXPEND. } \\
\text { Elasticity }\end{array}$ \\
\hline Food & $44 \%$ & & & & & & & & & & & & & & 1.48261 \\
\hline Non Food & $56 \%$ & & & & & & & & & & & & & & 1.84623 \\
\hline matooke & $5.7 \%$ & -1.030 & 0.235 & 0.085 & 0.056 & 0.079 & 0.386 & 0.470 & 0.086 & 0.099 & 0.353 & 0.033 & 0.0645 & -1.248 & 1.38963 \\
\hline maize & $9.2 \%$ & 0.389 & -0.686 & -0.072 & -0.089 & 0.905 & 0.414 & 0.752 & -0.293 & -0.082 & 0.041 & -0.934 & -0.474 & 1.029 & 1.06968 \\
\hline rice & $8.1 \%$ & 0.092 & -0.040 & -0.587 & 0.157 & 0.541 & -0.239 & 0.296 & 0.654 & 0.163 & 0.518 & 0.576 & 0.880 & -1.448 & 0.67178 \\
\hline sugar & $9.1 \%$ & 0.084 & -0.083 & 0.173 & -1.055 & 0.723 & -0.071 & -0.702 & 1.941 & 1.170 & 2.276 & 2.109 & -1.816 & -1.171 & 0.93375 \\
\hline cereal & $4.4 \%$ & 0.068 & 0.460 & 0.309 & 0.371 & -1.355 & -0.011 & -0.923 & -0.655 & -0.178 & 0.551 & -0.833 & -0.025 & 1.460 & 1.11284 \\
\hline oil & $4.2 \%$ & 0.275 & 0.194 & -0.133 & -0.034 & -0.006 & -1.089 & -0.314 & 0.195 & -0.085 & -0.055 & 0.113 & -0.049 & 0.380 & 0.95997 \\
\hline veg & $4.1 \%$ & 0.314 & 0.353 & 0.164 & -0.326 & -0.840 & -0.314 & -0.580 & -0.100 & 0.212 & 0.246 & -0.840 & -0.030 & 0.604 & 0.89534 \\
\hline meat & $14.1 \%$ & 0.268 & -0.487 & 1.143 & 7.896 & -2.033 & 0.651 & -0.338 & -1.589 & 0.419 & -0.381 & -1.311 & 1.587 & -1.289 & 1.19698 \\
\hline fish & $10.2 \%$ & 0.219 & -0.093 & 0.186 & 1.394 & -0.405 & -0.227 & 0.535 & 0.331 & -0.489 & 0.260 & 1.004 & 0.795 & -1.705 & 1.02799 \\
\hline dairy & $6.5 \%$ & 0.427 & 0.048 & 0.367 & 1.618 & 0.785 & -0.085 & 0.355 & -0.134 & 0.168 & -0.208 & -4.837 & -1.079 & -1.102 & 0.86276 \\
\hline beverage & $2.0 \%$ & -0.036 & -0.206 & 0.179 & 0.499 & -0.383 & 0.073 & -0.420 & -0.195 & 0.209 & -1.521 & -0.441 & 0.138 & 1.613 & 0.14338 \\
\hline alcohol & $9.6 \%$ & -0.061 & -0.534 & 1.234 & -2.004 & -0.094 & -0.088 & -0.048 & 1.051 & 0.755 & -1.582 & 0.894 & -0.234 & 0.121 & 0.76373 \\
\hline pulses & $5.6 \%$ & -1.157 & 0.727 & -1.119 & -1.692 & 1.108 & 0.499 & 0.824 & -2.418 & -2.469 & -1.005 & 1.451 & 0.164 & -1.460 & 1.40059 \\
\hline
\end{tabular}

Data source: UNHS 1999/2000

\section{Conclusions}

Ugandan households dwelling in urban settings differ significantly from their rural counterparts only in their consumption of fruits and vegetables. Low-income Ugandan households appear to substitute consumption within particular food groups, such as the starchy food group. For example, at low incomes, households substituted between cereal, matooke, maize, sugar, and rice, whereas at mean incomes, the household substitution is between cereal, rice, sugar, and maize.

The inclusion of matooke as a substitute for these starchy staples, especially for low-income consumers, leads us to conclude that there is greater substitution within the starchy food group. Ugandans with higher incomes consume more rice, fruits and vegetables, and soft beverages than their low-income counterparts. Low-income households, on their part, consumed more matooke, maize, and cereals, supporting previous studies in Africa that show higher income consumers shifting away from coarse grains, such as sorghum and millet. Significantly, households that are located in border areas consume greater quantities of matooke, sugar, oils, fruits and vegetables, dairy products, alcohol and pulses compared to interior districts.

Food purchases for households producing food (rural households) are more sensitive to price and income changes, especially as far as matooke is concerned. This sensitivity follows from these food-producing households being able to substitute home produced food for purchased food. As other studies have shown, home food production will lead to improved nutritional intake in Uganda. Because food and nutritional security is a major objective of the current government (NFNC, 2002), this study will also assist planners to identify policies that ensure adequate nutritional intake throughout Uganda.

Future research in this area needs to be done using latest Household Survey data that has been released by Uganda Bureau of Statistics (UBOS).

\section{References}

Asche, F. \& Wessells, C.R. (1997). On price indices in the almost ideal demand system. American Journal of Agricultural Economics, 79: 1182-1185.

Buse, A. (1994). Evaluating the linearized almost ideal demand system. American of Journal Agricultural Economics, 76: 781-793.

Deaton, A.,\&. Muellbauer, J.(1980b). An Almost Ideal Demand System. The American Economic Review, 70: 3312326.

Dorosh, P., del Ninno, C. and Sahn, D. E. (1994), Food aid and poverty alleviation in Mozambique. Cornell Food and Nutrition Policy Program. Working Paper 50. Cornell University, Ithaca, NY.

Green, R., \& Alston, J. M. (1990).Elasticities in AIDS models. American Journal of Agricultural Economics, May, $442-445$. 
Heckman, J. J. (1979) Sample selection bias as a specification error, Econometrica, 47, 153-161

Heien, D, and Wessells, C. R. (1990) Demand systems estimation with microdata A cencored regression approach, Journal of Business and Economic Statistics, (3): 365-371.

Heien, D., \& Durham, C., (1991). A Test of Habit Formation Hypothesis Using Household Data, Review of Economics and Statistics, 73, 189-199.

Intriligator, M., Bodkin, R., \& Hsiao, C. (1996). Econometric Models, Techniques and Applications, 2nd edition, London: Prentice Hall

Moschini, G. (1995). Units of measurement and the Stone index in demand system Estimation. American Journal of Agricultural Economics, 77 February, 63-68

Moschini, G. (1998). The semiflexible almost ideal demand system. European Economics Review 42: 346-364.

Nweke, F. I., Okorji, E.C. Njoku, J.E. and King, D. J. (1992). Elasticities of demand for

major food items in a root and tuber based food system. Research and Crop Management Monograph No. 11. International Institute of Tropical Agriculture.

Pollak, R.A. \& Wales, T.J. (1978). Estimation of complete demand systems from household budget data: the linear and quadratic expenditure systems. American Economic Review, 68(3): 348-359.

Pollak, R.A. \& Wales, T.J. 1981. Demographic variables in demand analysis. Econometrica, 49(6): 1533-1551.

Reardon, T., Delgado, C. and Thiombiano, T. (1992), Substitution of imported for

traditional cereals in household food consumption in Ouagadougou, Burkina Faso. Mimeo. International Food Policy Research Institute, Washington, DC.

Savadogo, K. and Brandt, J. A. (1988), Household foods demand in Burkina Faso: Implications for food policy, Agricultural Economics 2 (4), 345-364

Teklu, T. (1996). Food demand studies in Sub-Saharan: A survey of empirical evidence. Food Policy, 21, 479-496

Teutenberg, H. J. (1975). The General Relationship between Diet and Industrialization in European Diet from Preindustrilization to modern times, ed. E. and R. Foster, pp. 61-129. London: Harper and Row

UGANDA BUREAU OF STATISTICS (UBOS), (2005). Uganda National Household Survey 1999-2000, Entebbe, Uganda: UBOS

Working, H. (1943). Statistical laws of family expenditure. Journal of the American Statistical Association, 33: 43-56. 